\title{
ALOX12B promotes carcinogenesis in cervical cancer by regulating the PI3K/ERK1 signaling pathway
}

\author{
TAO JIANG ${ }^{1,2}$, BING ZHOU ${ }^{3}$, YUAN MENG LI $^{2}$, QUI YING YANG ${ }^{2}$, KAI JIA TU ${ }^{4}$ and LONG YU LI ${ }^{1,4}$ \\ ${ }^{1}$ Department of Gynecological Oncology, Jiangxi Medical College of Nanchang University, Nanchang, \\ Jiangxi 330031; Departments of ${ }^{2}$ Gynecology and ${ }^{3}$ Pathology, The Affiliated Jiujiang Hospital \\ of Nanchang University, Jiujiang, Jiangxi 332000; ${ }^{4}$ Department of Gynecological Oncology, \\ Jiangxi Maternal and Child Health Hospital, Nanchang, Jiangxi 330006, P.R. China
}

Received July 7, 2019; Accepted April 3, 2020

DOI: $10.3892 / 01.2020 .11641$

\begin{abstract}
Cervical cancer is a malignant disease and a threat to women's health worldwide. Surgical resection followed by radiotherapy or chemotherapy is the main treatment strategy for cervical cancer; however, patients with cervical cancer, especially those with late-stage disease, may not benefit from these traditional therapies, which results in poor clinical outcome. ALOX12B is a gene encoding lipoxygenase, and a mutation in ALOX12B was detected in lung and breast cancer. Furthermore, ALOX12B is essential to the proliferation of epidermoid carcinoma cells; however, the role of ALOX12B in cervical cancer has not been studied thus far, to the best of our knowledge. In the present study, the expression levels of ALOX12B were reduced in cervical cancer cells by lentiviral transfection, and it was found that both cell proliferation and clone formation ability were significantly reduced, and the cell cycle was blocked at $\mathrm{G}_{1}$ phase. Tumor growth was also suppressed in vivo in a xenograft tumor model, but the migration of tumor cells was not affected by ALOX12B. Subsequently, using western blotting, it was demonstrated that knockdown of ALOX12B decreased the expression levels of PI3K, MEK1, ERK1, $\mathrm{C}$-fos and cdc25. Meanwhile, overexpression of ALOX12B increased the expression levels of these five molecules. Conclusively, ALOX12B promoted cell proliferation in cervical cancer via regulation of the PI3K/ERK1 signaling pathway. The present study may improve our understanding of the molecular mechanisms underlying the function of ALOX12B in cervical cancer and inform new therapeutic strategies.
\end{abstract}

Correspondence to: Mrs. Long Yu Li, Department of Gynecological Oncology, Jiangxi Maternal and Child Health Hospital, 318 Bayi Street, Nanchang, Jiangxi 330006, P.R. China

E-mail: 1yli5919@163.com

Key words: ALOX12B, cervical cancer, PI3K, MEK1, C33A cells, Ca-Ski cells

\section{Introduction}

Cervical cancer is a common malignant tumor in women (1). According to a statistical report released in 2019, the number of estimated new cases was 13,170 , and the estimated cervical cancer-associated deaths will increase to 4,250 in the USA in 2019 (2). Meanwhile, in China, a total of 98,900 women were predicted to be diagnosed with cervical cancer, and $\sim 30,500$ cervical cancer-associated deaths were predicted in 2015 (3). Although the prognosis of cervical cancer is not dismal, the 5-year survival rate for patients with metastasisis $17 \%$, and it is $\sim 50 \%$ for those with regional cervical cancer (2); however, only $15 \%$ of patients are diagnosed with metastasis (2).

Currently, the primary therapy for regional cervical cancer is surgical resection followed by radiotherapy or chemotherapy, while palliative therapy for recurrent or metastatic cancer does not improve clinical survival time (4). The benefit from all these therapies is usually short lived and only effective in some patients (4). Recently, target therapy and immunotherapy have been introduced as new therapies for patients with cancer, particular for patients with late-stage cancer. For example, the chimeric antigen receptor T-cell (CAR-T) therapy drug KYMRIAH $^{\circledR}$ improved the prognosis in patients with acute lymphoblastic leukemia (5). However, the major issue regarding this novel therapy is that a critical protein has to be expressed in or on the surface of tumor cells. The single chain antibody fragment targeting CD19 molecule was essential for the clinical outcome as the CAR-T therapy drug recognized and bound to CD19 (5). The antibody drug KEYTRUDA ${ }^{\circledR}$ is another successful example for patients with cancer (6). PD-1 serves an inhibitory role in activation of $T$ cells, and KEYTRUDA $^{\circledR}$ blocks the inhibitory signaling via targeting PD-1, allowing T-cell activation. Therefore, elucidation of the molecular mechanism and identifying key molecules, such as PD-1,involved in tumor formation and progression is critical and necessary for new therapies.

Genetic mutations are important factors contributing to tumorigenesis (7). ALOX12B encodes a lipoxygenase, and is responsible for the conversion of arachidonic acid to $12 \mathrm{R}$-hydroxyeicosatetraenoic acid 8). The $A L O X 12 \mathrm{~B}$ gene is located at chromosome $17 \mathrm{p} 13.1$, and contains 15 exons (8). 
Lipoxygenases are reported to be associated with inflammation, skin disorders and tumorigenesis (9). Mutations in the $A L O X 12 B$ gene mainly result in non-bullous congenital ichthyosiform erythroderma $(10,11)$. ALOX12B has also been reported to inhibit immune cytolytic activity in breast and renal cell tumors (12), and single nucleotide polymorphisms (SNPs) in $A L O X 12 B$ are associated with increased risk of lung and breast cancer $(13,14)$. Agarwal et al $(15)$ reported that inhibition of ALOX12B directly reduced the proliferation of the vulvar epidermoid carcinoma A431 cell line. Hence, ALOX12B serves important roles in the carcinogenesis of tumors, but, to the best of our knowledge, there are no studies investigating the role of ALOX12B in cervical cancer.

The aim of the present study was to investigate the role of the $A L O X 12 B$ gene in cervical cancer by knocking down this gene in cervical cancer cells and systemically studying its role both in vitro and in vivo. Additionally, the underlying molecular mechanism of ALOX12B in cervical cancer was explored using western blot analysis.

\section{Materials and methods}

Cell culture. Human Ca-Ski and C33A cells were purchased from the American Type Culture Collection and cultured in DMEM (Gibco; Thermo Fisher Scientific, Inc.) containing $10 \%$ fetal bovine serum (Gibco; Thermo Fisher Scientific, Inc.) in a humidified atmosphere with $5 \% \mathrm{CO}_{2}$ at $37^{\circ} \mathrm{C}$. C33A cells are derived from HPV-negative cervical cancer, while $\mathrm{Ca}-\mathrm{Ski}$ cells are derived from HPV 16-positive cervical cancer (16).

Reverse transcription-quantitative (RT-q) PCR assay. RNA was extracted from tumor cells, including C33A and Ca-Ski cells, using an RNAeasy ${ }^{\mathrm{TM}}$ kit (Beyotime Institute of Biotechnology) according to the manufacturer's instructions. RNA was reverse transcribed into complementary DNA (cDNA) using $1 \mu \mathrm{g}$ RNA and PrimeScript ${ }^{\mathrm{TM}}$ 1st strand cDNA Synthesis kit (Takara Bio, Inc.). qPCR was carried out using SYBR Green mix (Shanghai Yeasen Biotechnology Co., Ltd.) using an ABI 7000 system (Applied Biosystems; Thermo Fisher Scientific, Inc.) and PrimeScript $^{\mathrm{TM}}$ RT Master mix (Takara Bio, Inc.). The thermocycling protocol was as follows: $95^{\circ} \mathrm{C}$ for $2 \mathrm{~min}$, followed by 40 cycles at $95^{\circ} \mathrm{C}$ for $15 \mathrm{sec}$ and $60^{\circ} \mathrm{C}$ for $15 \mathrm{sec}$. GAPDH was used as the internal control. The relative quantity of the target gene was calculated using the $2^{-\Delta \Delta C q}$ method (17). The primer sequences for each gene are shown in Table I.

Western blotting. Total protein was extracted using a protein extraction kit (Shanghai Yeasen Biotechnology Co., Ltd.) according to the manufacturer's protocol, and the protein concentration was determined using an ultraviolet spectrophotometer (Onedrop1000; Shanghai Genechem Co., Ltd.). A total of $10 \mu \mathrm{g}$ protein/lane was loaded on a $12 \%$ polyacrylamide gel, resolved using SDS-PAGE and transferred to polyvinylidene fluoride (PVDF) membranes (Sangon Biotech Co., Ltd.). The PVDF membrane was blocked with 5\% non-fat milk for $2 \mathrm{~h}$ at room temperature. Subsequently, the PVDF membrane was incubated with the corresponding primary antibody against the target protein overnight at $4^{\circ} \mathrm{C}$ followed by incubation with the secondary antibody at room temperature for $1 \mathrm{~h}$. The protein band was detected using a Beyo ECL Star kit
(Beyotime Institute of Biotechnology). The antibodies used were as follows: Anti-PI3K (ab70912; 1:100), anti-MEK1 (ab32091; 1:1,500), anti-ERK1 (ab32537; 1:600), anti-cdc25 (ab111830; 1:2,000) (all Abcam), anti-C-fos (554C1a; 1:500; Santa Cruz Biotechnology, Inc.), anti-ALOX12B (PA5-23608; 1:800; Invitrogen; Thermo Fisher Scientific, Inc.), anti-GAPDH (ab181602; 1:10,000; Abcam), goat anti-mouse IgG antibody (ab97035; 1:2,000) and goat anti-rabbit IgG antibody (ab7090; 1:5,000) (both Abcam).

Package of lentiviral vector carrying small hairpin RNA(shRNA) targeting ALOX12B and transduction. Three shRNA fragments targeting the $A L O X 12 B$ gene were designed, synthesized and transferred into the expression vector pPLK-GFP (pPLK-shALOX12B-GFP). The sequences of each shRNA fragment are shown in Table II. In parallel, a non-targeting sequence was used as the negative control (NC). Subsequently, a lentiviral vector carrying shALOX12B (LvshALOX12B) was prepared as follows: $10 \mu \mathrm{g}$ pspax 2 plasmid (Invitrogen; Thermo Fischer Scientific, Inc.), $5 \mu \mathrm{g}$ pMD2.G plasmid (Invitrogen; Thermo Fisher Scientific, Inc.) and $15 \mu \mathrm{g}$ pPLK-shALOX12B-GFP were transferred into 293 T cells (RanYanBio Co., Ltd.) and cultured in DMEM containing 10\% FBS (both Gibco; Thermo Fisher Scientific, Inc.) at $37^{\circ} \mathrm{C}$ with $5 \% \mathrm{CO}_{2}$ for $72 \mathrm{~h}$. Subsequently, the supernatant was collected, and the lentiviral particles were concentrated from the supernatant by centrifugation at $14,000 \mathrm{xg}$ at $4^{\circ} \mathrm{C}$ for $40 \mathrm{~min}$.

Cervical cancer cells were treated with LvshALOX12B or NC (scrambled) for $6 \mathrm{~h}$ and then cultured for $72 \mathrm{~h}$. The transduction efficiency was detected under a fluorescence microscope (magnification, x100; Nikon Corporation). The knockdown efficiency of ALOX12B was determined by qPCR assay as aforementioned.

Construction of plasmid expressing ALOX12B and transfection. The coding sequence for ALOX12B (NM_001139.3) was synthesizedandclonedintothe pcDNA3.1 vector(Addgene,Inc.). The recombinant plasmid pcDNA3.1-ALOX12B (to overexpress ALOX12B) was confirmed by DNA sequencing. Subsequently, thepcDNA3.1-ALOX12B plasmid was transfected into tumor cells using Lipofectamine reagent (40802ES02; Shanghai Yeasen Biotechnology Co., Ltd.). The transfection efficiency was detected using western blotting for detection of ALOX12B expression levels, as aforementioned. The time interval between transfection and subsequent experimentation was $48 \mathrm{~h}$.

Cell proliferation assay using Cell Counting Kit-8 (CCK-8). A total of 6,000 lentiviral-infected C33A or Ca-Ski cells/well were seeded into a 96-well plate. After $24,48,72$ or $96 \mathrm{~h}$ of culture, $10 \mu \mathrm{l}$ CCK-8 (Beyotime Institute of Biotechnology) was added into each well and cells were cultured at $37^{\circ} \mathrm{C}$ with $5 \% \mathrm{CO}_{2}$ for another $4 \mathrm{~h}$ according to the manufacturer's protocol. Subsequently, the absorbance at $450 \mathrm{~nm}$ was determined using a microplate reader. Each assay was repeated independently at least three times.

Colony forming assay. A total of $100 \mathrm{C} 33 \mathrm{~A}$ or Ca-Ski cells/well were seeded into a 24 -well plate. After 15 days, the cells 
Table I. Sequence of the primers used for quantitative PCR.

\begin{tabular}{ccc}
\hline Gene name & \multicolumn{1}{c}{ Sequence, 5'-3' } & $\begin{array}{c}\text { Fragment } \\
\text { size, bp }\end{array}$ \\
\hline $\begin{array}{c}\text { ALOX12B } \\
\text { Forward }\end{array}$ & GCCTGTTGGACTGCAAACATT & 196 \\
Reverse & GTGACGGGGAACTTGTCTGG & \\
GAPDH & & 217 \\
Forward & TCATCATCTCTGCCCCCTCT & \\
Reverse & GGGCCATCCACAGTCTTCTG & \\
\hline
\end{tabular}

Table II. shRNA target sequences for ALOX12B.

\begin{tabular}{ll}
\hline Name & \multicolumn{1}{c}{ Target sequence, 5'-3' } \\
\hline shALOX12B-1 & GATACACCGTCCAGATCAAT \\
shALOX12B-2 & GCACGCGGATCCCAGACAA \\
shALOX12B-3 & CCGATATGTCACTATAGTCAT \\
NC & GTTCTCCGAACGTGTCACGTT \\
\hline
\end{tabular}

$\mathrm{NC}$, negative control; sh, small hairpin.

on the plate were fixed with $4 \%$ paraformaldehyde at room temperature for $30 \mathrm{~min}$ and stained with $0.1 \%$ crystal violet at room temperature for $15 \mathrm{~min}$.The colony number was counted manually under a light microscope (magnification, $\mathrm{x} 40$ ). Each assay was repeated independently at least three times.

Wound healing assay. A total of $2 \times 10^{4} \mathrm{C} 33 \mathrm{~A}$ orCa-Skicells/well were seeded into a 24 -well plate and cultured to $90 \%$ confluence. Next, a $10-\mu 1$ sterile tip was used to make a scratch in the middle of each well. The debris was washed with PBS, and fresh DMEM without FBS was added. After $24 \mathrm{~h}$, the plate was observed, and images were captured under a light microscope (magnification, x100), and the width of each scratch was recorded. Each assay was repeated independently at least three times.

Cel cycle analysis. A total of $1 \times 10^{5} \mathrm{C} 33 \mathrm{~A}$ or $\mathrm{Ca}-\mathrm{Ski}$ cells treated with lentiviral vector for $72 \mathrm{~h}$ were harvested and washed with cold PBS followed by fixing in 70\% ethyl alcohol. Next, $0.5 \mathrm{ml}$ $1 \mathrm{X}$ staining buffer (Beyotime Institute of Biotechnology) with $10 \mu 1$ propidium iodide (PI) reagent (Beyotime Institute of Biotechnology) was used to resuspend the tumor cells, which were then cultured for $30 \mathrm{~min}$ at $37^{\circ} \mathrm{C}$. The cell cycle distribution was analyzed using fluorescence-activated cell sorting (Beckman Coulter, Inc.).

In vivo tumor growth assay. Nanchang Royo Biotech Co., Ltd. performed the following experiments: 20 female BALB/c-nu mice (6 weeks old; $20-30 \mathrm{~g}$ ) were obtained from Shanghai SLAC Laboratory Animal Co., Ltd. and divided equally into NC and shALOX12B groups. Mice were kept in groups of 4 or 5 in individual cages and provided with sterilized food and water ad libitum. Mice were maintained in conditions with a 12 -h light-dark cycle at $22^{\circ} \mathrm{C}$ and $55 \%$ humidity. After in vitro culture, $\sim 2 \times 10^{7} \mathrm{C} 33 \mathrm{~A}$ cancer cells were inoculated subcutaneously into the right flank. Tumor growth was monitored consecutively for 35 days. Subcutaneous tumor volume (V) was measured twice a week and calculated as follows: $\mathrm{V}=\left(\mathrm{LxW}^{2}\right) / 2$, where $\mathrm{L}$ is the length and $\mathrm{W}$ is the width of the tumor. At the 35th day, mice were anesthetized according to the guidelines involving the use of diethyl ether approved by the Laboratory Animal Ethics Committee of Nanchang Royo Biotech Co., Ltd. (approval no. RYE2019011104); a sterile gauze soaked in $99.5 \%$ diethyl ether was placed in a $500 \mathrm{ml}$ beaker and mice were placed into the beaker for $5 \mathrm{~min}$. Subsequently, the mice were sacrificed by cervical dislocation. If the tumor volume was $>1,500 \mathrm{~mm}^{3}$ or if ulcers occurred, the study was terminated prematurely.

Statistical analysis. All data are displayed as the mean \pm standard deviation, and were analyzed using SPSS version 16.0 (SPSS Inc.). The difference between two groups was compared using an unpaired Student's t-test. The differences among multiple groups were compared using a one-way ANOVA followed by Tukey's post hoc test. $\mathrm{P}<0.05$ was considered to indicate a statistically significant difference.

\section{Results}

Effective ALOX12B-knockdown in cervical cancer C33A cells. LvshALOX12B was used to transfect C33A cells, and the transfection efficiency was monitored by GFP expression. As shown in Fig. 1A, the transfection efficiency was high after transduction for $72 \mathrm{~h}$, according to GFP expression. Subsequently, qPCR technology was used to determine the expression levels of the $A L O X 12 B$ gene in C33A cells. qPCR analysis demonstrated that the knockdown efficiency of each shRNA was $>75 \%$ compared with that of the NC (Fig. 1B). The knockdown efficiency of shALOX12B-3 was $~ 90 \%$; therefore, shALOX12B-3 was chosen for functional analysis.

Knockdown of ALOX12B inhibits the proliferation and colony formation of C33A cells. A CCK-8 assay was designed to detect the effect of shALOX12B-3 on the proliferation of C33A and Ca-Ski cells. As shown in Fig. 2A and B, the proliferation rate of C33A treated with shALOX12B-3 was $69 \%$ compared with that of the NC group, while the proliferation rate was $76 \%$ in Ca-Ski cells after transfection for $96 \mathrm{~h}$. Colony forming ability also reflects the proliferative potential. The relative colony formation rate in the NC group was significantly higher compared with that of the shALOX12B-3 group in C33A cells ( $\mathrm{P}<0.05$; Fig. 2Cand D). For Ca-Ski cells, the relative colony formation rate in the $\mathrm{NC}$ group was also significantly higher compared with that of the shALOX12B-3 group $(\mathrm{P}<0.05$; Fig. $2 \mathrm{C}$ and $\mathrm{D})$. These results suggested that ALOX12B participates in the proliferation and growth of cervical cancer cells.

ALOX12B is not essential for cell migration in cervical cancer. In general, cancer cells have a marked migration ability in the majority of cancers (18). A wound healing assay was performed to determine the effect of shALOX12B-3 on the migration of cervical cancer cells. However, no significant 

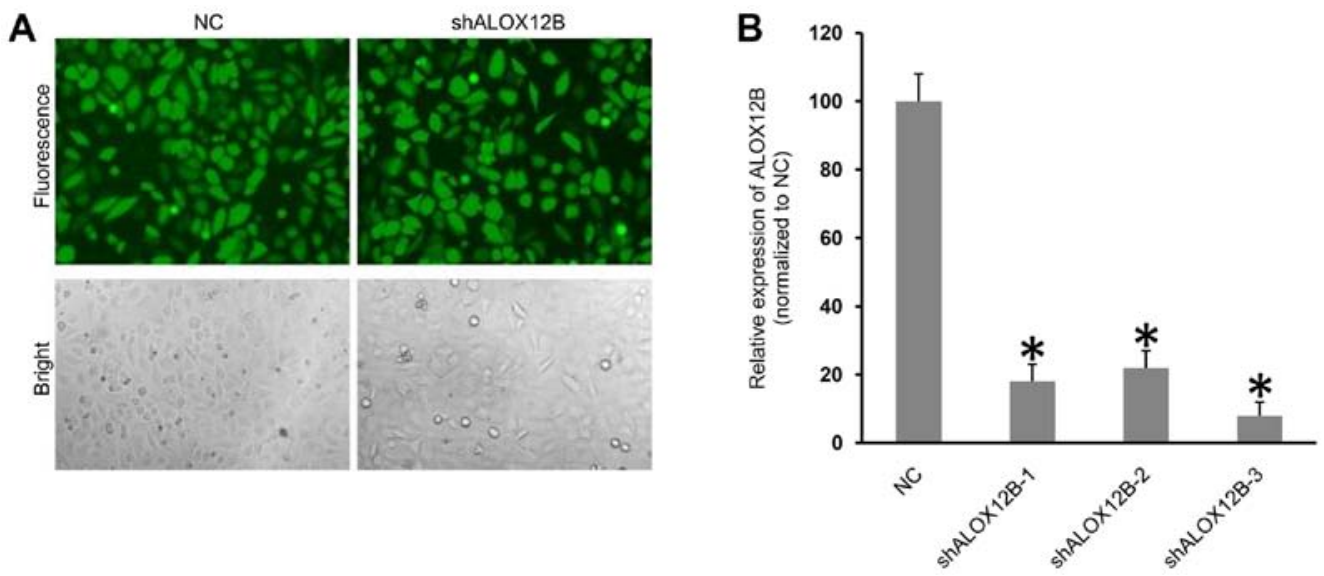

Figure 1. ALOX12B-knockdown in cervical cancer cells. (A) GFP expression of C33A transfected with the lentiviral expression vector carrying shALOX12B or NC after $72 \mathrm{~h}$. Magnification, x100. (B) Quantitative PCR analysis of ALOX12B expression levels after lentiviral vector transfection for $72 \mathrm{~h}$. "P $<0.05$. sh, small hairpin; NC, negative control; GFP, green fluorescent protein.
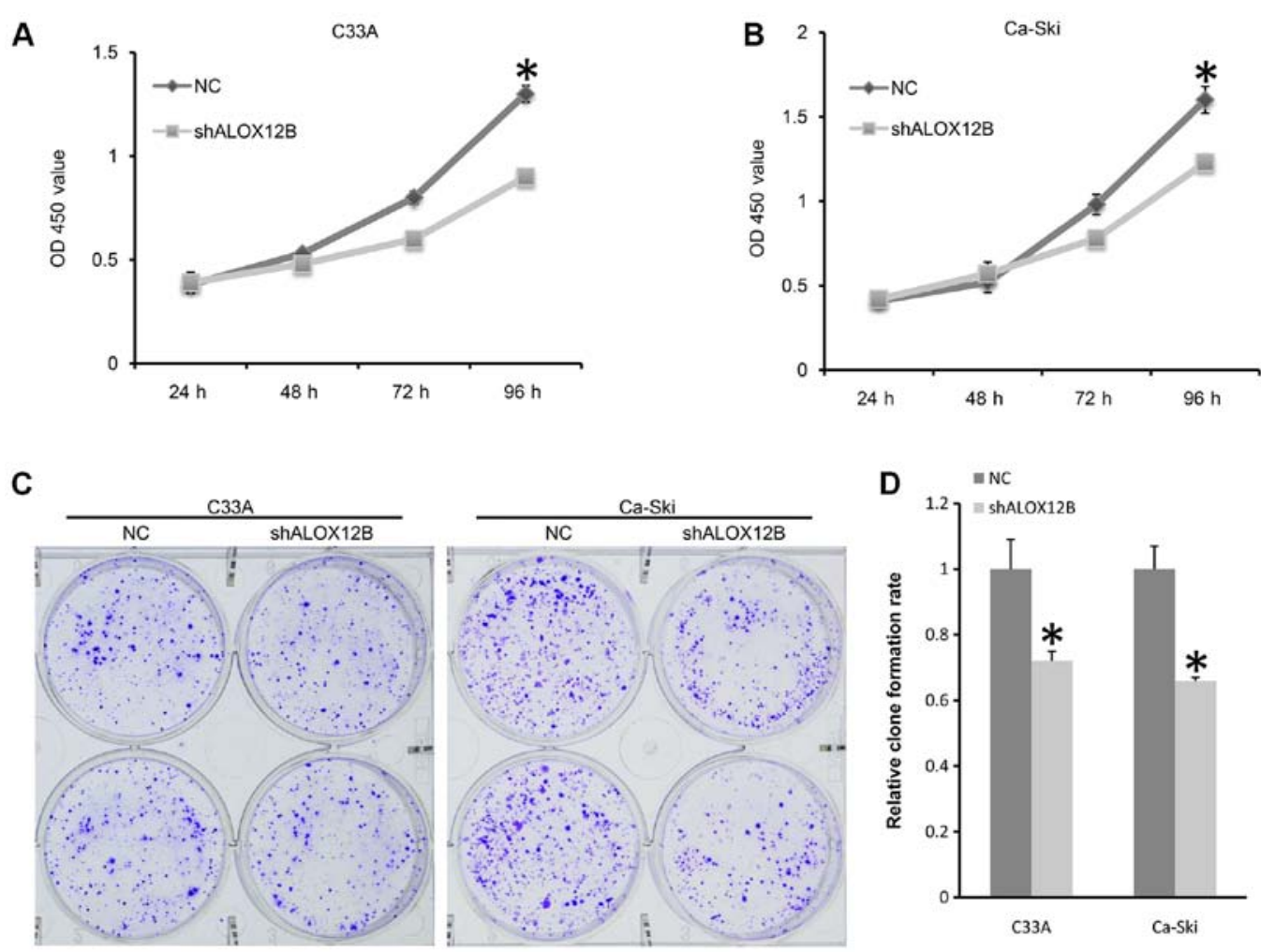

Figure 2. Knockdown of ALOX12B inhibits cell proliferation and colony formation. The proliferation of (A) C33A and (B) cells Ca-Ski was inhibited when the ALOX12B levels were decreased following knockdown. (C) The colony forming ability of C33A cells and Ca-Ski cells was inhibited after ALOX12B-knockdown. The top plates are replicates of the bottom plates. (D) Quantitative analysis of colony formation in C33A and Ca-Ski cells upon ALOX12B-knockdown or transfection with NC. ${ }^{*} \mathrm{P}<0.05$. sh, small hairpin; NC, negative control; OD, optical density.

difference was observed at $24 \mathrm{~h}$ between the $\mathrm{NC}$ group and the shALOX12B-3 group in C33A or Ca-Ski cells (Fig. 3A and B). The 48-h time point was also assessed in the wound healing assay, but no significant differences were observed, as the relative migration rate in the $\mathrm{NC}$ group was equivalent to that in the shALOX12B group (data not shown). Therefore, it is possible that ALOX12B may not regulate cell migration in cervical cancer.

Cell cycle transition is suppressed by ALOX12B-knockdown. To detect the effect of shALOX12B-3 on cell cycle in cervical cancer, C33A and Ca-Ski cells were stained with PI dye after treatment with shALOX12B-3 and NC. As shown in Fig. 4A and B, the cell cycle was arrested at the $\mathrm{G}_{1}$ phase after shALOX12B was knocked down. The ratio of $\mathrm{C} 33 \mathrm{~A}$ cells in the $\mathrm{G}_{1}$ phase increased from 37.4 to $55.7 \%$ (NC vs. shALOX12B). By contrast, no significant difference was observed in $\mathrm{Ca}-\mathrm{Ski}$ cells. The phase distribution in $\mathrm{Ca}-\mathrm{Ski}$ cells showed no significant difference between the shALOX12B-3 and NC groups (Fig. 4C and D). This might be attributed to biological variation between different cells. 
A
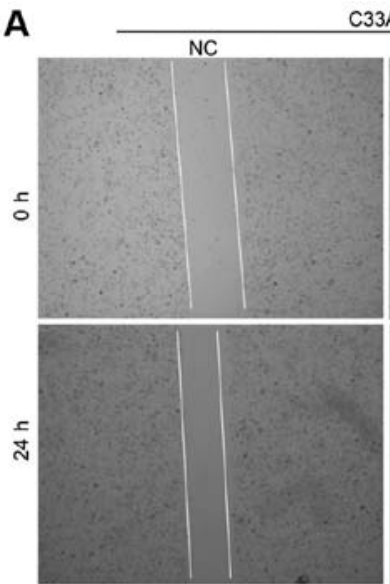

$33 \mathrm{~A}$
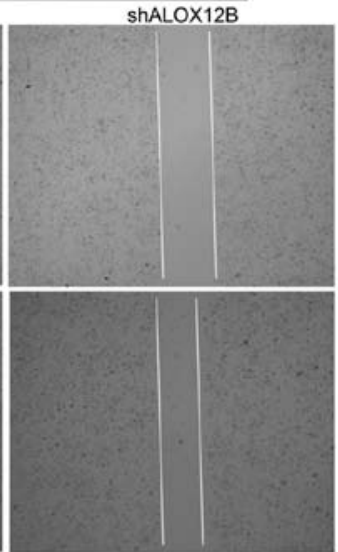

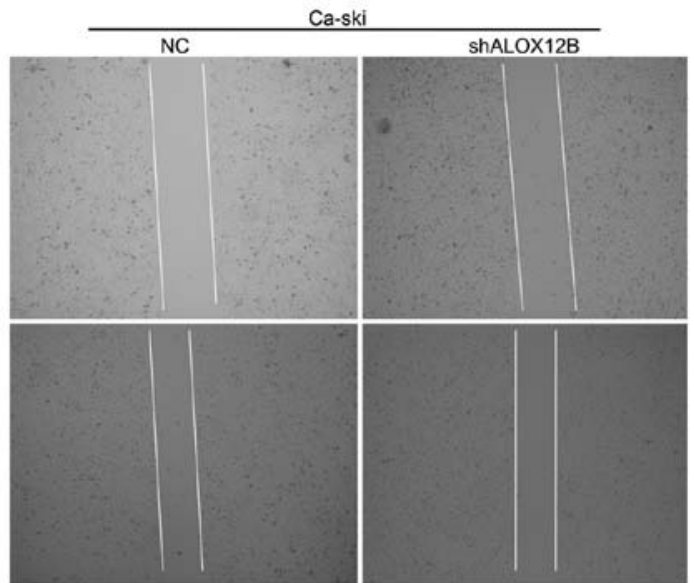

B

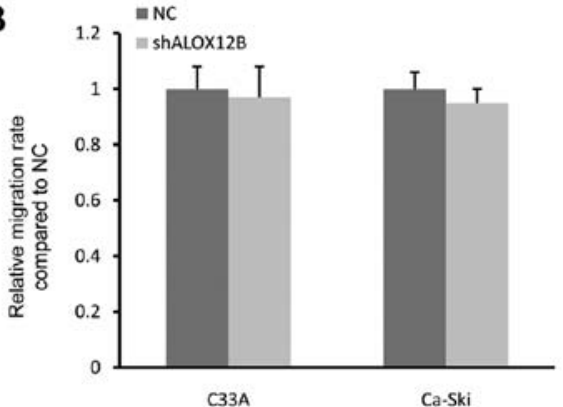

Figure 3. ALOX12B does not affect cell migration in cervical cancer. (A) Wound healing assay demonstrated that the knockdown of ALOX12B had no obvious impact on cell migration. (B) Data analysis of the relative cell migration rate of C33A and Ca-Ski cells transfected with shALOX12B or NC. sh, small hairpin $\mathrm{NC}$, negative control.

A

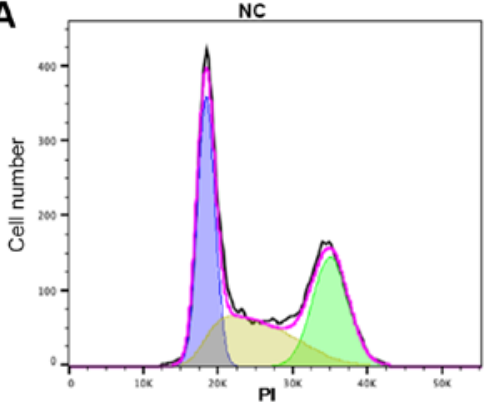

C

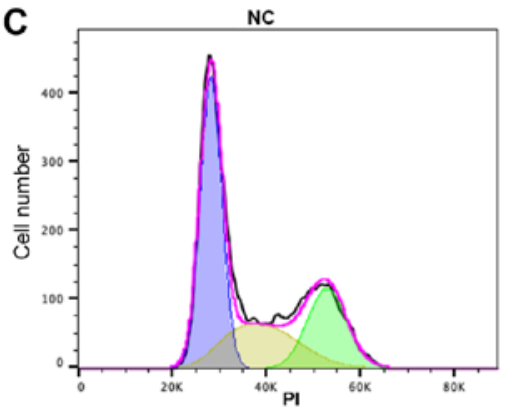

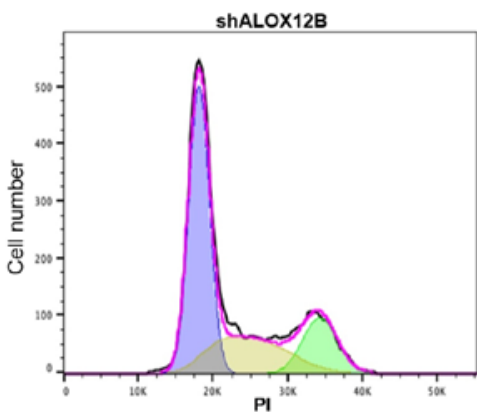

B
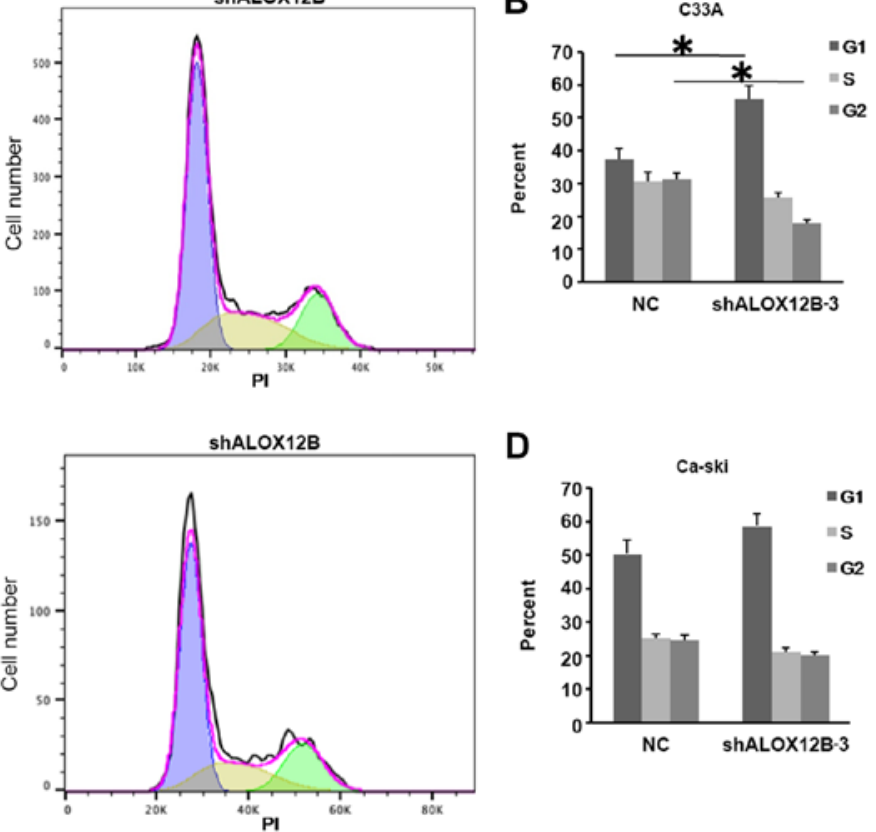

D

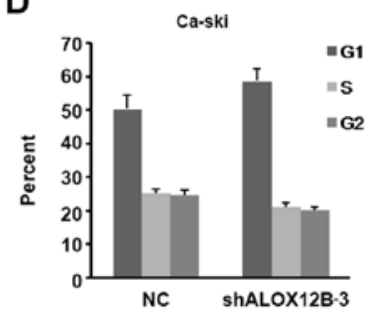

Figure 4. ALOX12B regulates cell cycle transition in cervical cancer. (A) FACs analysis demonstrating that knockdown of ALOX12B arrested cell cycle in the $G_{1}$ phase in C33A cells. (B) The difference between cells in $G_{1}$ phase in the NC group and cells in $\mathrm{G}_{1}$ phase in the shALOX12B group was significant. The difference between cells in $\mathrm{G}_{2}$ phase in the $\mathrm{NC}$ group and cells in $\mathrm{G}_{2}$ phase in the shALOX12B group was also significant. (C) FACs analysis demonstrating that knockdown of ALOX12B did not affect the cell cycle in Ca-Ski cells. (D) No significant differences in the cell cycle distribution in Ca-Ski cells were found after ALOX12B-knockdown. "P<0.05. sh, small hairpin; NC, negative control; FACs, fluorescent-activated cell sorting.

Knockdown of ALOX12B delays tumor growth in a xenograft mouse model. Since ALOX12B contributed to the cell proliferation and growth of $\mathrm{C} 33 \mathrm{~A}$ cells, and regulated the cell cycle distribution, it was hypothesized that ALOX12B 
A
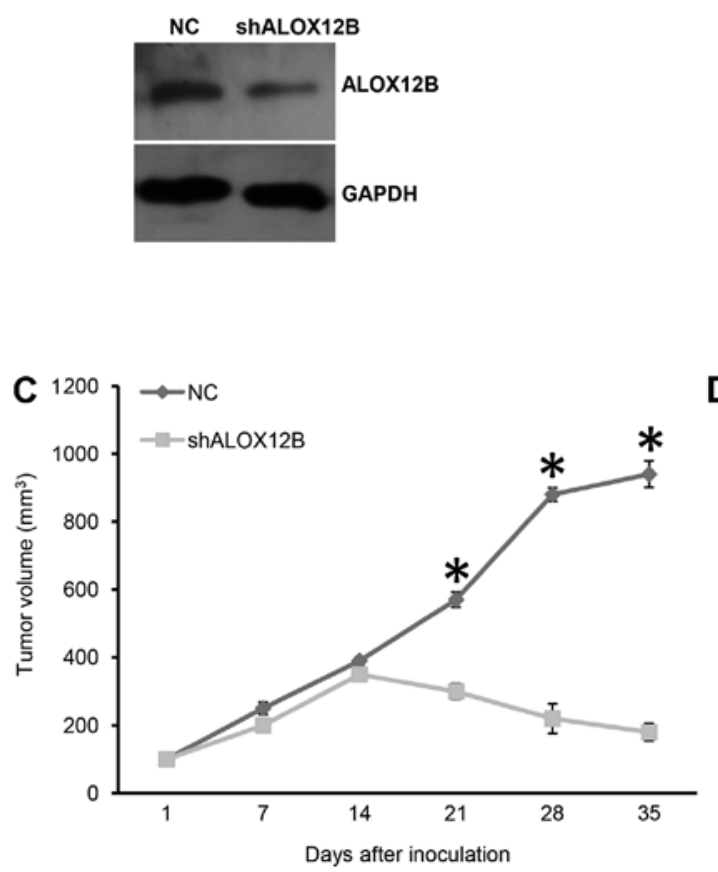

B

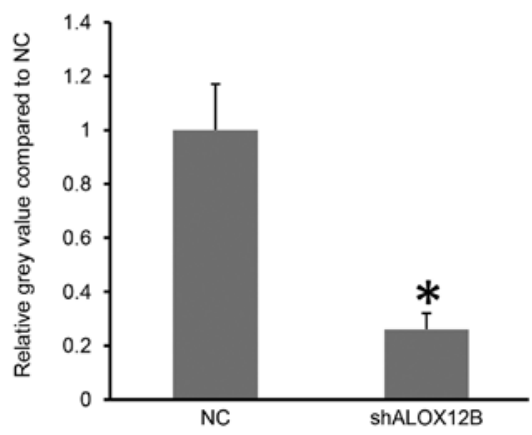

D

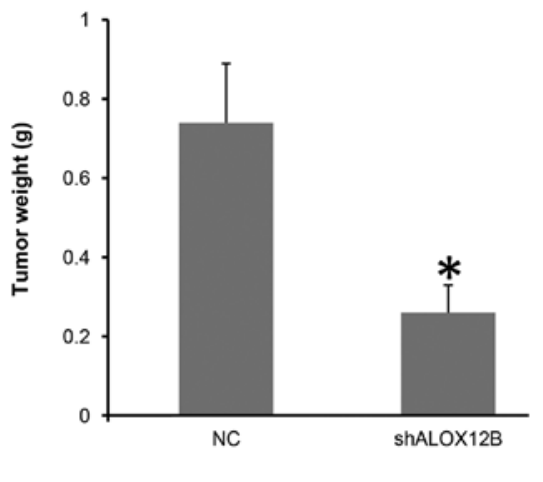

Figure 5. Knockdown of ALOX12B suppresses tumor growth in vivo. (A and B) Western blotting confirmed the knockdown of ALOX12B in vivo. (C) The tumor volume of mice in the shALOX12B group was significantly smaller than that of mice in the NC group. (D) The tumor weight of mice in shALOX12B group was significantly smaller than that of mice in the NC group. ${ }^{*} \mathrm{P}<0.05$. sh, small hairpin; NC, negative control.

could regulate tumor growth in vivo. C33A cells transfected with shALOX12B-3 showed a significant slower growth in a mouse xenograft model $(\mathrm{P}<0.05$; data not shown). As shown in Fig. 5A and B, the knockdown of ALOX12B was confirmed by western blotting. It was demonstrated that the tumor volume in the shALOX12B-3 group was significantly smaller compared with that of the $\mathrm{NC}$ group (180 vs. $940 \mathrm{~mm}^{3} ; \mathrm{P}<0.05$; Fig. 5C). The tumor weight in the shALOX12B-3 group was also significantly lighter compared with that of the $\mathrm{NC}$ group (0.26 vs. 0.74g; P<0.05; Fig. 5D). As expected, ALOX12B was essential for in vivo tumor growth of cervical cancer.

ALOX $12 B$ regulates the PI3K/ERK1 signaling pathway in cervical cancer. As a novel gene, there is little information regarding the signaling pathway regulated by ALOX12B. In several types of cancer, important signaling pathways regulating cell proliferation include $\mathrm{PI} 3 \mathrm{~K}$, Wnt, mTOR and MAPK (19-21). The expression levels of these molecules were analyzed using western blotting. As shown in Fig. 6A and B, after knockdown of ALOX12B in C33A cells, the expression levels of PI3K, MEK1, ERK1, C-fos and cdc25 were significantly reduced $(\mathrm{P}<0.05)$. By contrast, overexpression of ALOX12B in cells treated with the plasmid pcDNA-ALOX12B significantly increased the expression levels of PI3K, MEK1, ERK1, C-fos and cdc25 ( $\mathrm{P}<0.05$; Fig. 6A and $\mathrm{C}$ ). In vivo, knockdown of ALOX12B also significantly reduced the expression of PI3K, MEK1, ERK1, C-fos and cdc25 ( $\mathrm{P}<0.05$; Fig. 6A and D). PI3K/ERK1signaling serves important roles in several types of cancer (19); therefore, it is possible that ALOX12B promotes the proliferation of cervical cancer cells by regulating the PI3K/ERK1 signaling pathway.

\section{Discussion}

Cervical cancer is a malignant disease affecting women worldwide, and poses a great threat to life and life quality $(2,3)$. According to the most recent report by the American Association for Cancer Research, both the incidence and death rate of patients with cervical cancer has remained steady in recent years (1). This could be attributed to the failure of traditional therapies such as surgery, chemotherapy and radiotherapy. This high death rate indicates that some patients with cervical cancer, particularly those who have relapsed or have metastasis, do not benefit sufficiently from such therapies (3). In the past decade, a series of new biotherapies have been developed, including immunotherapy and targeted therapy. For example, antibody drugs and cell immunotherapy have greatly improved the survival of patients with cancer $(4,5)$. However, to harness the potential clinical benefit of these therapies, the underlying molecular mechanisms of a particular cancer type need to be clarified to prevent treatment failure.

The majority of cervical cancer cases are caused by HPV infection, which is most commonly transmitted through sexual activity (22). However, genome sequencing technology has revealed that genetic and epigenetic factors also serve important roles during the tumorigenesis of cervical cancer (23). Several genes have been reported to be associated with cervical cancer. For example, Chang et al (24) found that high POUF3 expression accelerated the progression of cervical cancer. Heterogeneity is common in almost all types of cancer (25); therefore, it is difficult to determine the function of a single dominant gene in a specific type of cancer. 

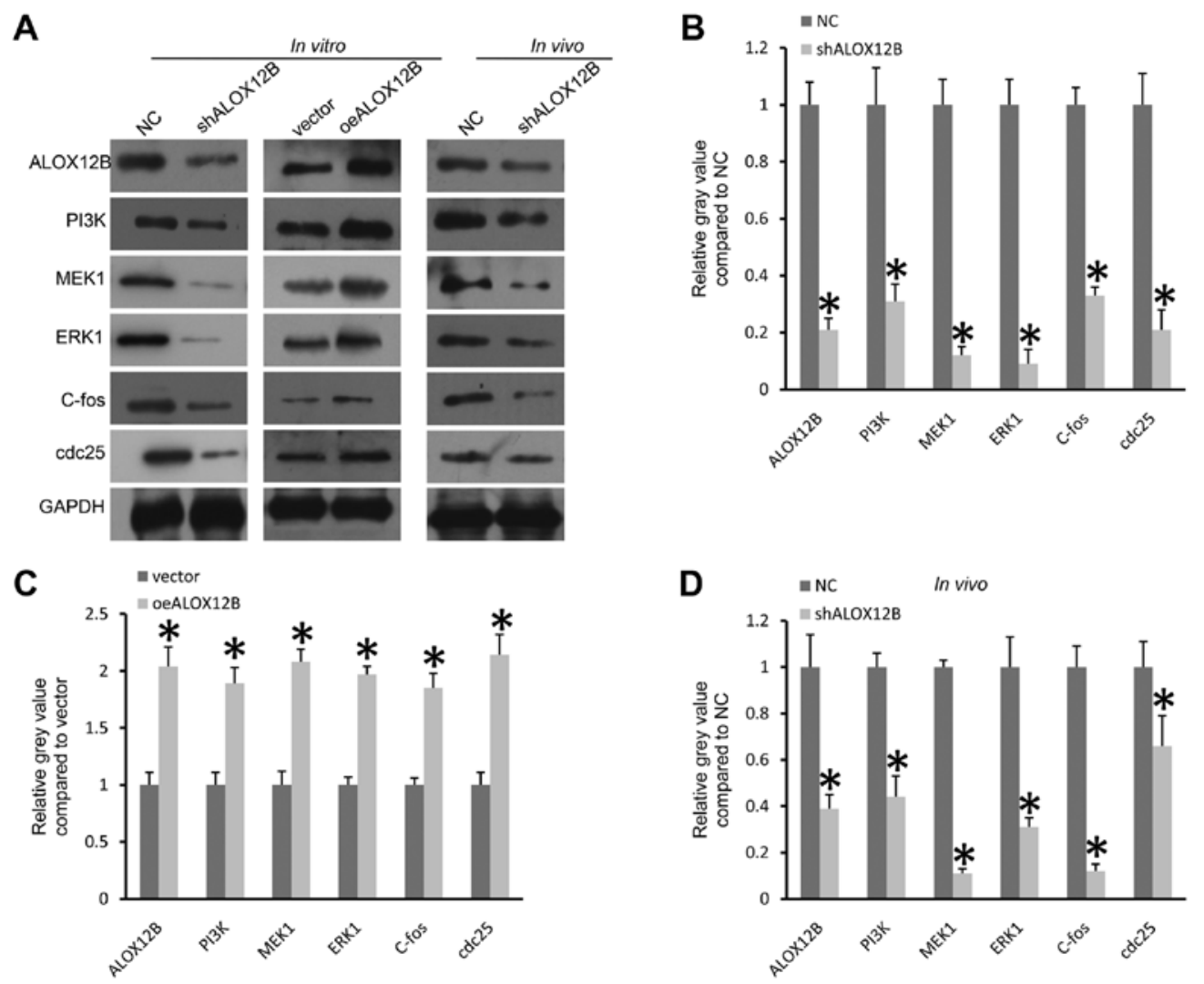

Figure 6. ALOX12B regulates the expression levels of PI3K/ERK1 signaling molecules. (A and B) Knockdown of ALOX12B decreased the expression levels of PI3K, MEK1, ERK1, C-fos and cdc25 in C33A cells. (A and C) Overexpression of ALOX12B in C33A cells increased the expression levels of PI3K, MEK1, ERK1, C-fos and cdc25. (A and D) Knockdown of ALOX12B decreased the expression levels of PI3K, MEK1, ERK1, C-fos and cdc25 in tumor tissues in the xenograft tumor model. GAPDH was used as the internal control. "P<0.05. sh, small hairpin; NC, negative control; oe, overexpressed.

To the best of our knowledge, the present study demonstrated for the first time that ALOX12B may serve an oncogenic role in cervical cancer. The in vitro data suggested that ALOX12B contributed to cell proliferation in cervical cancer. Normally, cells display an inhibitory effect when they contact each other; however, tumor cells are known to have no contact-inhibitory characteristics and can readily proliferate (16). An accelerated cell cycle is often synchronized with cell proliferation and is common in tumor cells. As expected, knockdown of ALOX12B resulted in cell cycle arrest in the $\mathrm{G}_{1}$ phase in C33A cells. This was consistent with the proliferation-promoting role of ALOX12B in cervical cancer. However, little difference in cell cycle was observed in Ca-Ski cells. This may be due to the heterogeneity of tumor cells. Infection with HPV was a major cause of cervical cancer, and different strains of HPV caused expression of checkpoint molecules controlling the cell cycle, such as p53 and p105 $(16,26,27)$.

Metastasis is another common phenomenon affecting patients with cancer (18). Generally speaking, wound healing assays are often used to reflect the aggressive ability of tumor cells in vitro (28); however, ALOX12B-knockdown did not affect the migration ability of cervical cancer cells in the present study. It is possible that ALOX12B solely regulates cell proliferation in cervical cancer. The mouse xenograft model bearing C33A cells further demonstrated that ALOX12B was essential for tumor cell growth in vivo. These data suggested that ALOX12B promoted the progression of cervical cancer. In previous studies, ALOX12B was shown to be involved in the progression of lung, breast and epidermoid cancer (13-15); thus, the present study may further improve our understanding of the role of ALOX12B in cancer. However, additional experiments are necessary to confirm the role of ALOX12B in promoting the progression of cervical cancer. Although ALOX12B displayed little effect on cell migration in vitro, it may be useful to analyze the effect of ALOX12B on the metastasis of cervical cancer cells in vivo and to investigate the expression patterns of ALOX12B in tumor versus normal tissues.

ALOX12B is a lipoxygenase that is critical for the synthesis of 12R-hydroxyeicosatetraenoic acid (10). The lipoxygenase family of proteins is large and comprises dozens of members. Different lipoxygenases were shown to be associated with inflammation and tumorigenesis via different signaling pathways (9). For example, ALOX15 suppresses inflammation by inhibiting the IL-6/STAT3 signaling pathway in colorectal cancer (29). Additionally, lipoxygenase 5-LOX and 12-LOX have been revealed to be essential for cell proliferation in pancreatic cancer (30). The most commonly reported alteration concerning ALOX12B was SNP (8-14). Only Agarwal et al (15) demonstrated that ALOX12B modulated the ERK and PI3K-Akt signaling pathways in A431 cells. As ALOX12B was shown to regulate cell proliferation in cervical cancer in the present study, the proliferation-related signaling molecules in C33A cells were determined. It was found that ALOX12B could regulate the expression levels of PI3K, MEK1, ERK1, C-fos and cdc25.PI3K is known to be an important 
gene in the proliferation of tumor cells, and abnormal activation of PI3K has been shown in a number of cancer types, including cervical cancer (31). MEK1 is a mitogen-activated protein kinase and is often involved in crosstalk with the PI3K signaling pathway. For example, MEK1 transmits signals from PI3K to ERK1, and activates target genes such as C-fos and cdc25 (32). Activation of the PI3K/ERK1 signaling pathway has been observed in several types of cancer and has been shown to promote tumorigenesis (33). C-fos and cdc25 are two common genes that promote tumor progression $(27,34)$. In addition, ALOX12B was shown to regulate PI3K and ERK signaling pathways in epidermoid cancer cells (15); therefore, it is possible that ALOX12B regulates PI3K/ERK1 signaling in cervical cancer.

In summary, the present study demonstrated that ALOX12B promoted cell proliferation via regulation of the PI3K/ERK1 signaling pathway in cervical cancer. These results may provide the basis for future targeting of ALOX12B in cervical cancer treatment. However, the clinical significance of ALOX12B in cervical cancer remains unclear. The association of ALOX12B with survival and with clinicopathological factors in patients was not elucidated in the present study. Additionally, the way in which ALOX12B affects the PI3K signaling pathway requires further investigation. Another limitation is that the present study only used a couple of cell lines, and therefore the role of the ALOX12B gene should be investigated in more cell lines, which may further support the present results. Therefore, more work is required to further support the role of ALOX12B in cervical cancer and to clarify the link between ALOX12B and PI3K/ERK1. The expression pattern of ALOX12B in a large cohort of clinical tumor tissues and its association with clinicopathological factors should be investigated to confirm its clinical significance.

\section{Acknowledgements}

Not applicable.

\section{Funding}

No funding was received.

\section{Availability of data and materials}

The datasets used and/or analyzed during the current study are available from the corresponding author on reasonable request.

\section{Authors' contributions}

TJ and LYL conceived and designed the study. QYY and YML conducted the literature review and interpreted the data. BZ and TJ analyzed the data. TJ wrote the manuscript. KJT, TJ, BZ and YML performed the experiments. All authors read and approved the final manuscript.

\section{Ethics approval and consent to participate}

The present study was approved by the Laboratory Animal Ethics Committee of Nanchang Royo Biotech Co., Ltd. (approval no. RYE2019011104).

\section{Patient consent for publication}

Not applicable.

\section{Competing interests}

The authors declare that they have no competing interests.

\section{References}

1. Johnson CA, James D, Marzan A and Armaos M: Cervical Cancer: An Overview of Pathophysiology and Management. Semin Oncol Nurs 35: 166-174, 2019.

2. Siegel RL, Miller KD and Jemal A: Cancer statistics, 2019. CA Cancer J Clin 69: 7-34, 2019.

3. Chen W, Zheng R, Baade PD, Zhang S, Zeng H, Bray F, Jemal A, Yu XQ and He J: Cancer statistics in China, 2015. CA Cancer J Clin 66: 115-132, 2016.

4. Liontos M, Kyriazoglou A, Dimitriadis I, Dimopoulos MA and Bamias A: Systemic therapy in cervical cancer: 30 years in review. Crit Rev Oncol Hematol 137: 9-17, 2019.

5. Leahy AB, Elgarten CW, Grupp SA, Maude SL and Teachey DT: Tisagenlecleucel for the treatment of B-cell acute lymphoblastic leukemia. Expert Rev Anticancer Ther 18: 959-971, 2018.

6. Kwok G, Yau TC, Chiu JW, Tse E and Kwong YL: Pembrolizumab (Keytruda). Hum Vaccin Immunother 12: 2777-2789, 2016.

7. Fang J, Zhang $\mathrm{H}$ and Jin S: Epigenetics and cervical cancer: From pathogenesis to therapy. Tumour Biol 35: 5083-5093, 2014.

8. Krieg P, Marks F and Fürstenberger G: A gene cluster encoding human epidermis-type lipoxygenases at chromosome 17p13.1: Cloning, physical mapping, and expression. Genomics 73: 323-330, 2001.

9. Mashima R and Okuyama T: The role of lipoxygenases in pathophysiology; new insights and future perspectives. Redox Biol 6: 297-310, 2015.

10. Kurban M, Shimomura Y, Bahhady R, Ghosn S, Kibbi AG and Christiano AM: Nonsense mutation in the ALOX12B gene leads to autosomal recessive congenital ichthyosis in a Lebanese family. J Eur Acad Dermatol Venereol 24: 232-234, 2010.

11. Rodríguez-Pazos L, Ginarte M, Vega A and Toribio J: Autosomal recessive congenital ichthyosis. Actas Dermosifiliogr 104: 270-284, 2013.

12. Rooney MS, Shukla SA, Wu CJ, Getz G and Hacohen N: Molecular and genetic properties of tumors associated with local immune cytolytic activity. Cell 160: 48-61, 2015.

13. Shen M, Vermeulen R, Rajaraman P, Menashe I, He X, Chapman RS, Yeager M, Thomas G, Burdett L, Hutchinson A, et al: Polymorphisms in innate immunity genes and lung cancer risk in Xuanwei, China. Environ Mol Mutagen 50: 285-290, 2009.

14. Lee JY, Park AK, Lee KM, Park SK, Han S, Han W, Noh DY, Yoo KY, Kim H, Chanock SJ, et al: Candidate gene approach evaluates association between innate immunity genes and breast cancer risk in Korean women. Carcinogenesis 30: 1528-1531, 2009.

15. Agarwal S, Achari C, Praveen D, Roy KR, Reddy GV and Reddanna P: Inhibition of $12-\mathrm{LOX}$ and COX-2 reduces the proliferation of human epidermoid carcinoma cells (A431) by modulating the ERK and PI3K-Akt signalling pathways. Exp Dermatol 18: 939-946, 2009.

16. Bernard B,Fest T,Prétet JL and Mougin C: Staurosporine-induced apoptosis of HPV positive and negative human cervical cancer cells from different points in the cell cycle. Cell Death Differ 8: 234-244, 2001.

17. Livak KJ and Schmittgen TD: Analysis of relative gene expression data using real-time quantitative PCR and the $2(-\Delta \Delta \mathrm{C}(\mathrm{T}))$ Method. Methods 25: 402-408, 2001.

18. Hanahan D and Weinberg RA: Hallmarks of cancer: The next generation. Cell 144: 646-674, 2011.

19. Khajah MA, Mathew PM and Luqmani YA: Inhibitors of PI3K/ERK1/2/p38 MAPK Show Preferential Activity Against Endocrine-Resistant Breast Cancer Cells. Oncol Res 25: 1283-1295, 2017.

20. Taciak B, Pruszynska I, Kiraga L, Bialasek M and Krol M: Wnt signaling pathway in development and cancer. J Physiol Pharmacol 69: 69, 2018.

21. Zeng H: mTOR signaling in immune cells and its implications for cancer immunotherapy. Cancer Lett 408: 182-189, 2017. 
22. Tsikouras P, Zervoudis S, Manav B, Tomara E, Iatrakis G, Romanidis C, Bothou A and Galazios G: Cervical cancer: Screening, diagnosis and staging. J BUON 21: 320-325, 2016.

23. Le Gallo M, Lozy F and Bell DW: Next-Generation Sequencing. Adv Exp Med Biol 943: 119-148, 2017.

24. Chang S, Sun L and Feng G: SP1-mediated long noncoding RNA POU3F3 accelerates the cervical cancer through miR-127-5p/FOXD1. Biomed Pharmacother 117: 109133, 2019.

25. Fasterius E, Uhlén M and Al-Khalili Szigyarto C: Single-cell RNA-seq variant analysis for exploration of genetic heterogeneity in cancer. Sci Rep 9: 9524, 2019.

26. Crook T, Wrede D, Tidy JA, Mason WP, Evans DJ and Vousden KH: Clonal p53 mutation in primary cervical cancer: Association with human-papillomavirus-negative tumours. Lancet 339: 1070-1073, 1992.

27. Thomas M, Pim D and Banks L: The role of the E6-p53 interaction in the molecular pathogenesis of HPV. Oncogene 18: 7690-7700, 1999.

28. Justus CR, Leffler N, Ruiz-Echevarria M and Yang LV: In vitro cell migration and invasion assays. J Vis Exp 88: 51046, 2014.

29. Tian R, Zuo X, Jaoude J, Mao F, Colby J and Shureiqi I: ALOX15 as a suppressor of inflammation and cancer: Lost in the link. Prostaglandins Other Lipid Mediat 132: 77-83, 2017.
30. Ding XZ, Tong WG and Adrian TE: Cyclooxygenases and lipoxygenases as potential targets for treatment of pancreatic cancer. Pancreatology 1: 291-299, 2001.

31. Bossler F, Hoppe-Seyler K and Hoppe-Seyler F: PI3K/AKT/mTOR Signaling Regulates the Virus/Host Cell Crosstalk in HPV-Positive Cervical Cancer Cells. Int J Mol Sci 20: 20, 2019.

32. De Luca A, Maiello MR, D'Alessio A, Pergameno $M$ and Normanno N: The RAS/RAF/MEK/ERK and the PI3K/AKT signalling pathways: Role in cancer pathogenesis and implications for therapeutic approaches. Expert Opin Ther Targets 16 (Suppl 2): S17-S27, 2012.

33. Milde-Langosch K: The Fos family of transcription factors and their role in tumourigenesis. Eur J Cancer 41: 2449-2461, 2005.

34. Brenner AK, Reikvam H, Lavecchia A and Bruserud $\varnothing$ : Therapeutic targeting the cell division cycle 25 (CDC25) phosphatases in human acute myeloid leukemia - the possibility to target several kinases through inhibition of the various CDC25 isoforms. Molecules 19: 18414-18447, 2014.

(i) (9) This work is licensed under a Creative Commons Attribution-NonCommercial-NoDerivatives 4.0 International (CC BY-NC-ND 4.0) License. 